\title{
Towards Ontological Foundations for the Conceptual Modeling of Events
}

\author{
Giancarlo Guizzardi ${ }^{1}$, Gerd Wagner ${ }^{2}$, Ricardo de Almeida Falbo ${ }^{1}$, \\ Renata S.S. Guizzardi ${ }^{1}$, João Paulo A. Almeida ${ }^{1}$ \\ ${ }^{1}$ Ontology and Conceptual Modeling Research Group (NEMO), \\ Federal University of Espírito Santo (UFES), Brazil \\ \{ gguizzardi, falbo, rguizzardi, jpalmeida \}@inf.ufes.br \\ ${ }^{2}$ Institute of Informatics, \\ Brandenburg University of Technology, Germany \\ G.Wagner@tu-cottbus.de
}

\begin{abstract}
In recent years, there has been a growing interest in the application of foundational ontologies, i.e., formal ontological theories in the philosophical sense, to provide a theoretically sound foundation for improving the theory and practice of conceptual modeling. In this paper, we present advances on our research on the ontological foundations of conceptual modeling by addressing the concept of events. We present a foundational ontology of events (termed UFO$B)$ together with its axiomatization in first-order logic. Moreover, we report on an implementation of UFO-B using the computational logic language Alloy, and discuss its consistency, validation and possible uses.
\end{abstract}

Keywords: Ontological Foundations for Conceptual Modeling, Formal Ontology, Ontology of Events

\section{Introduction}

In recent years, there has been a growing interest in the application of formal ontological theories to provide a theoretically sound foundation for improving the theory and practice of conceptual modeling. A number of efforts have shown the benefits of ontology-based techniques in the evaluation and redesign of conceptual modeling languages [1-3], in making explicit the deep semantics of natural language [4], and, when the ontology is described formally, as a basis for validation [5] and automated reasoning [6].

The success of such ontology-based efforts depends on the availability of comprehensive ontological foundations, which have been the object of research in the past decades by several groups, under the banners of upper-level, top-level and foundational ontologies $[3,7,8]$. In this paper, we focus on a philosophically and cognitively well-founded reference ontology called UFO (Unified Foundational Ontology), which has been part of a long term research program on foundations for conceptual modeling [3]. UFO has been developed based on theories from Formal Ontology, Philosophical Logics, Philosophy of Language, Linguistics and Cognitive Psychology. 
The core categories of UFO have been completely formally characterized in [3]. This core fragment has been employed to analyze structural conceptual modeling constructs such as object types and taxonomic relations, associations and relations between associations, roles, properties, datatypes and weak entities, and parthood relations among objects. Moreover, it has been used to analyze, redesign and integrate reference conceptual models in a number of complex domains such as, for instance, Petroleum and Gas, Telecommunications, Software Engineering and Bioinformatics ${ }^{1}$.

Despite a significant number of positive results in this enterprise, the focus on the core fragment of this foundational ontology has been on addressing structural conceptual modeling concepts, as opposed to dynamic ones (i.e., events and related notions). This trend can also be found in other foundational ontologies. For instance, in the BWW ontology, the treatment of the notion of events is rather minimal, i.e., an event is taken simply as a transition between states in the lawful state space of a thing [2]. However, given the importance of the notion of events for enterprise modeling [1], knowledge representation and reasoning [6], information systems engineering [9] and the semantic web [10], we argue that a widely applicable foundation for conceptual modeling requires a fuller account of the ontological notion of events, which is the subject of this paper.

The remainder of this paper is organized as follows: Section 2 presents a background on the Unified Foundational Ontology (UFO) as a context for the results developed here; Section 3 presents the main contribution of this paper, namely, a foundational ontology of events termed UFO-B with its full formal characterization; Section 4 discusses evaluation of this ontology and shows an illustrative proof of concept; Section 5 discusses related work in the literature, and, finally, Section 6 presents final considerations and a discussion on the implications of UFO-B to the practice of conceptual modeling.

\section{Background: The Unified Foundational Ontology (UFO)}

Like other foundational ontologies, such as DOLCE [7] and GFO [8], UFO makes a fundamental distinction between enduring and perduring individuals (henceforth called endurants and events respectively). Classically, this distinction can be understood in terms of their behavior w.r.t. time. Endurants are said to be wholly present whenever they are present, i.e., they are in time, in the sense that if we say that in circumstance $\mathrm{c}_{1}$ an endurant $e$ has a property $\mathrm{P}_{l}$ and in circumstance $\mathrm{c}_{2}$ the property $\mathrm{P}_{2}$ (possibly incompatible with $\mathrm{P}_{1}$ ), it is the very same endurant $e$ that we refer to in each of these situations. Examples of endurants are a house, a person, the Moon, an amount of sand. For instance, we can say that an individual John weights $80 \mathrm{~kg}$ at $\mathrm{c}_{1}$ but $68 \mathrm{~kg}$ at $\mathrm{c}_{2}$. Nonetheless, we are in these two cases referring to the same individual.

Events (also called perdurants) are individuals composed of temporal parts. They happen in time in the sense that they extend in time accumulating temporal parts. Examples of events are a conversation, a football game, a symphony execution, a

${ }^{1}$ Related publications can be found in $\underline{\text { http://nemo.inf.ufes.br/en/publications }}$ 
birthday party, or a particular business process. Whenever an event is present, it is not the case that all its temporal parts are present.

Among the categories of endurants, UFO makes a distinction between objects and tropes (see Fig.1). Objects are existentially independent entities. Examples include ordinary mesoscopic objects such as an individual person, a car, Alan Turing and The Rolling Stones. Tropes are endurants that are existentially dependent on other entities (termed their bearers) in the way in which, for example, an electrical charge can exist only in some conductor. We define a particular relation of existential dependence between tropes and their bearers called inherence [3]. Inherence is a type of specific constant and functional existential dependence relation. Intuitively, we have that, for instance, the headache of John can only exist if John exists and cannot be dependent on anyone but John. The notion of tropes employed here includes both what are termed qualities (e.g., the color of an eye, the atomic number of an atom) as well as dispositions [11] (e.g. the fragility of a glass, the electrical conductivity of a material).

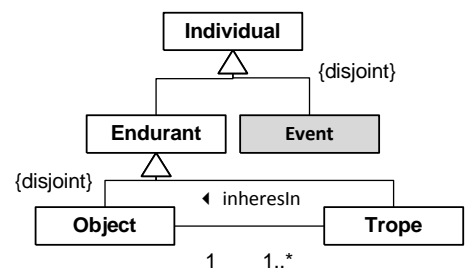

Fig.1. A fragment of the Unified Foundational Ontology (UFO)

\section{UFO-B: An Ontology of Events}

The theory of UFO-B presented here has been fully axiomatized in standard predicate calculus and in the computational logic languages Alloy [12]. In the classical firstorder logic axiomatization, we use a restricted quantification mechanism, which correspond to Frege's analysis of restricted quantification, i.e., $(\forall x: T \boldsymbol{A})$ is simply a shortcut for $(\forall x \boldsymbol{T}(\boldsymbol{x}) \rightarrow \boldsymbol{A})$ as well as $(\exists x: T \boldsymbol{A})$ is simply a shortcut for $(\exists x \boldsymbol{T}(\boldsymbol{x}) \wedge \boldsymbol{A})$.

In the following five subsections, we elaborate on different viewpoints of this ontology of events, namely: the mereological structure of events (Section 3.1); the participations of objects in events (Section 3.2); temporal ordering of events (Section 3.3); events as mappings from situations to situations in reality (Section 3.4); and events as manifestations of object's dispositions (Section 3.5).

\subsection{Event Mereology}

One first aspect of events is that events may be composed of other events. Take for instance the event $e$ : the murder of Caesar. This event can be further decomposed into sub-events, namely: $\mathrm{e}_{1}$ : the attack on Caesar, $\mathrm{e}_{2}$ : Caesar's death. Event $\mathrm{e}_{1}$ can, in turn, be decomposed in the events $\mathrm{e}_{11}$ : Caesar's restraining by the conspirators, and $\mathrm{e}_{12}$ : the stabbing of Caesar by Brutus. Events can be atomic or complex, depending on their mereological structure. Whilst atomic events have no proper parts, complex events are aggregations of at least two disjoint events, see Fig.2. 


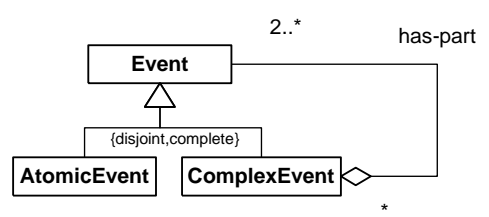

Fig.2. Complex and atomic events

As for any mereological theory, we assume the has-part relation between events to be a strict partial order (axioms M3-M5). Moreover, we assume an atomistic mereology for events, i.e., in our theory there are events which cannot be further decomposed and, hence, are atomic w.r.t. the parthood relation [13]. We define that atomic and complex events form a (disjoint, complete) partition (axioms M1 and M2).

\begin{tabular}{|c|c|}
\hline M1 & $\forall \mathrm{e}:$ Event AtomicEvent(e) $\leftrightarrow \neg \exists \mathrm{e}^{\prime}:$ Event has-part(e,e') \\
\hline M2 & $\forall \mathrm{e}:$ Event ComplexEvent $(\mathrm{e}) \leftrightarrow \neg$ AtomicEvent(e) \\
\hline M3 & $\forall \mathrm{e}:$ ComplexEvent $\neg$ has-part(e,e) \\
\hline M4 & $\forall$ e,e':ComplexEvent has-part(e,e') $\rightarrow \neg$ has-part(e',e) \\
\hline M5 & $\forall$ e,e':ComplexEvent, e': Event has-part(e,e' $) \wedge$ has-part(e',e'’ $) \rightarrow$ has-part(e,e' $)$ \\
\hline
\end{tabular}

As discussed in [13], axioms M3-M5 do not suffice to characterize a parthood relation, since a number of other non-mereological relations are also partial order relations (e.g., causality, less than, temporal ordering). An additional axiom is necessary to comprise what is known as Minimum Mereology (MM), namely, the so-called Weak Supplementation Axiom (WSP). Intuitively, WSP states that if an object is complex then it must have at least two disjoint (i.e., non-overlapping) parts (M6). The notion of mereological overlap is defined in (M7). We here also subscribe to the view defended in [13] that an Event Mereology should commit to what is termed an Extensional Mereology. By including what is termed the Strong Supplementation Axiom in MM (M8), we obtain the mereological equivalent of the extensionality principle of set theory, i.e., two events are the same if they are composed of the same parts (M9).

\begin{tabular}{|c|c|}
\hline M6 & $\begin{array}{l}\forall \mathrm{e}: \text { ComplexEvent, e':Event has-part(e,e') } \rightarrow \\
\exists \mathrm{e} ": \text { Event has-part(e,e'") } \wedge \neg \text { overlaps(e',e') }\end{array}$ \\
\hline M7 & 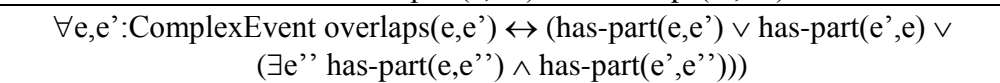 \\
\hline M8 & 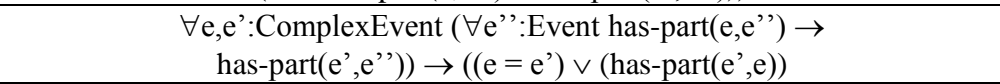 \\
\hline M9 & $\forall \mathrm{e}, \mathrm{e}^{\prime}:$ ComplexEvent $\left(\mathrm{e}=\mathrm{e}^{\prime}\right) \leftrightarrow\left(\forall \mathrm{e}^{\prime \prime}:\right.$ Event has-part(e,e') $\leftrightarrow$ has-part(e',e' $\left.)\right)$ \\
\hline
\end{tabular}

\subsection{On the Participation of Objects in Events}

Events are ontologically dependent entities in the sense that they existentially depend on objects in order to exist. As previously discussed, events can be either (mereologically) atomic or complex. An atomic event is said to be directly existentially dependent on an object. This relation (termed here dependsOn) is the perdurant counterpart of the inherence relation between tropes and their bearers, i.e., dependsOn is a specific constant dependence relation [7]. Moreover, as inherence, dependsOn (defined for atomic events) is a functional relation (P1). A complex event is also an 
existentially dependent entity. Due to the extensionality principle of the event mereology adopted here, we have that a Complex Event e' is existentially dependent on all its proper parts and, indirectly, to the objects these proper parts depend on.

The existential dependence of events on objects provides for an orthogonal way of partitioning complex events. Besides the mereological decomposition of events discussed in section 3.2, we can partition a complex event e' by separating each part of this event which is existentially dependent on each of its participants. Let us take as an example, the complex event $e_{12}$ : the stabbing of Caesar by Brutus. This event can be decomposed into the events $e_{\text {Brutus }}, e_{\text {Caesar }}, e_{\text {dagger }}$, which depend on (in the technical sense above) Brutus, Caesar and the dagger, respectively. We here term the portion of an event which depends exclusively on a single object a participation. As an orthogonal way of partitioning events, participations can be atomic or complex.

In the sequel, we present an axiomatization of the notion of participation as put forth here. Firstly, we define the notion exclusive dependence in the following manner: an atomic event is always exclusively dependent on a single object $o(\mathrm{P} 2)$; a complex event $e^{\prime}$ is exclusively dependent on an object $o$ iff all its proper parts exclusively depend on $o(\mathrm{P} 3)$.

\begin{tabular}{|c|c|}
\hline P1 & $\forall$ e:AtomicEvent $\exists$ !o:Object dependsOn(e,o) \\
\hline $\mathrm{P} 2$ & $\forall$ e:AtomicEvent, o:Object excDepends(e,o) $\leftrightarrow$ dependsOn(e,o) \\
\hline P3 & $\begin{array}{l}\forall \mathrm{e}: \text { ComplexEvent, o:Object excDepends }(\mathrm{e}, \mathrm{o}) \leftrightarrow \\
\left(\forall \mathrm{e}^{\prime}: \text { Event hasPart }\left(\mathrm{e}, \mathrm{e}^{\prime}\right) \rightarrow \text { excDependsOn }\left(\mathrm{e}^{\prime}, \mathrm{o}\right)\right)\end{array}$ \\
\hline P4 & $\forall$ e:Event Participation(e) $\leftrightarrow \exists$ !o:Object excDepends $(\mathrm{e}, \mathrm{o})$ \\
\hline P5 & $\forall \mathrm{o}:$ Object, p:Participation participationOf(p,o) $\leftrightarrow$ excDepends(p,o) \\
\hline
\end{tabular}

Fig. 3 summarizes the results of this subsection and depicts these two aspects on which events can be analyzed, namely, as entities with certain mereological structures, and as ontologically dependent entities consisting of a number of individual participations. As expressed in figure 3, the relations of exclusively depends on, participation of and the notion of participation itself are all derived notions (derived from the relations of parthood and existential dependence). Nonetheless, making explicit the notion of participation is of great importance from an ontological as well as conceptual point of view. We shall return to this point in sections 5 and 6 .

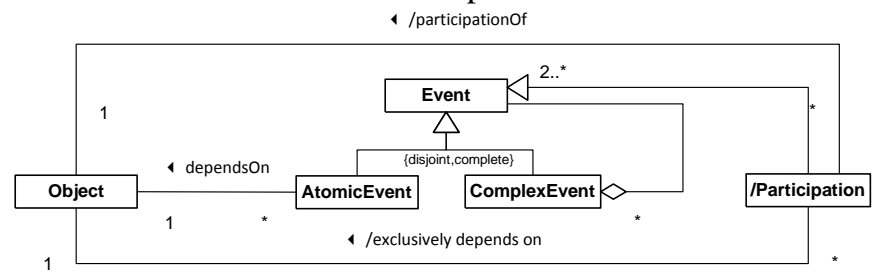

Fig.3. Complex Events as Sums of Object's Participations

\subsection{Temporal Relations between Events}

As in [7], we have that all spatial properties of events are defined in terms of the spatial properties of their participants. In contrast, all temporal properties of objects are defined in terms of the events they participate in. Analogous to what has been discussed elsewhere for objects [3], also the temporal properties of events are represent- 
ed by corresponding temporal attributes taking their values from a suitable quality structure (or property value space). As discussed in depth in [3], quality structures are geometrical/topological structures associated to a property of a given type organizing the possible values that an object can assume for that property.

By decoupling a property from its value space, UFO allows for a number of alternative spaces to be associated with a given property. For instance, the property color of an object can be associated with a RGB cubic space as well as with the HSV color spindle [3]. Here, we take the same approach for specifying the temporal properties of events. For instance, we can have a quality structure "composed of" time intervals and time intervals themselves to be "composed of" time points. Alternatively, we can have time specified by sui generis entities, such as the Chronoids and Time Boundaries of GFO [8]. Moreover, we can have a model of time that admit intervals that are delimited by begin and end points as well as open intervals, continuous and noncontinuous intervals, intervals with and without duration (instants). Finally, we can have models that allow for a diversity of temporal structures, such as linear, branching, parallel and circular time. Here, for illustration purposes and in order to avoid making unnecessary ontological commitments at this point, we adopt a simple but useful temporal structure: a linear order of time points. Each event is associated with two values: a begin-point and an end-point and time points are strictly ordered by a precedes relation. The set of temporal relations between two events corresponds to the well-known time interval relations proposed by Allen [14].

In the sequel, following [14], we present an axiomatization of these temporal Allen relations (T7-T13). In addition, we also define the linear time point space as a total order (T1-T4) and define two auxiliary functions for the begin-point and end-point of an event (T5-T6). Finally, we state that the temporal extent of an event (improperly) includes the temporal extent of all its (proper) parts (T14).

\begin{tabular}{|c|c|}
\hline T1 & $\forall \mathrm{t}:$ TimePoint $\neg$ precedes $(\mathrm{t}, \mathrm{t})$ \\
\hline $\mathrm{T} 2$ & $\forall \mathrm{t}, \mathrm{t}^{\prime}:$ TimePoint precedes $\left(\mathrm{t}, \mathrm{t}^{\prime}\right) \rightarrow \neg$ precedes $\left(\mathrm{t}^{\prime}, \mathrm{t}\right)$ \\
\hline T3 & $\forall \mathrm{t}, \mathrm{t}^{\prime}, \mathrm{t}^{\prime \prime}: \operatorname{TimePoint} \operatorname{precedes}\left(\mathrm{t}, \mathrm{t}^{\prime}\right) \wedge \operatorname{precedes}\left(\mathrm{t}^{\prime}, \mathrm{t}^{\prime \prime}\right) \rightarrow \operatorname{precedes}\left(\mathrm{t}, \mathrm{t}^{\prime}{ }^{\prime}\right)$ \\
\hline T4 & $\forall \mathrm{t}, \mathrm{t}^{\prime}:$ TimePoint $\left(\mathrm{t} \neq \mathrm{t}^{\prime}\right) \rightarrow \operatorname{precedes}\left(\mathrm{t}, \mathrm{t}^{\prime}\right) \vee \operatorname{precedes}\left(\mathrm{t}^{\prime}, \mathrm{t}\right)$ \\
\hline T5 & $\forall \mathrm{e}:$ Event $\exists$ !t:TimePoint, $\exists ! \mathrm{t}^{\prime}:$ TimePoint $(\mathrm{t}=$ begin-point $(\mathrm{e})) \wedge\left(\mathrm{t}{ }^{\prime}=\right.$ end-point $\left.(\mathrm{e})\right)$ \\
\hline T6 & $\forall$ e:Event precedes( begin-point(e), end-point(e)) \\
\hline $\mathrm{T} 7$ & $\forall \mathrm{e}, \mathrm{e}^{\prime}:$ Event before(e,e') $\leftrightarrow$ precedes( end-point(e), begin-point(e')) \\
\hline T8 & $\forall \mathrm{e}, \mathrm{e}^{\prime}:$ Event meets $\left(\mathrm{e}, \mathrm{e}^{\prime}\right) \leftrightarrow\left(\right.$ end-point $(\mathrm{e})=$ begin-point $\left.\left(\mathrm{e}^{\prime}\right)\right)$ \\
\hline T9 & $\begin{array}{c}\forall \mathrm{e}, \mathrm{e}^{\prime}: \text { Event overlaps }\left(\mathrm{e}, \mathrm{e}^{\prime}\right) \leftrightarrow \leftrightarrow \operatorname{precedes}\left(\text { begin-point }(\mathrm{e}), \text { begin-point }\left(\mathrm{e}^{\prime}\right)\right) \wedge \\
\text { precedes }\left(\text { begin-point }\left(\mathrm{e}^{\prime}\right), \text { end-point }(\mathrm{e})\right) \wedge \operatorname{precedes}\left(\text { end-point }(\mathrm{e}), \text { end-point }\left(\mathrm{e}^{\prime}\right)\right)\end{array}$ \\
\hline T10 & $\begin{array}{c}\forall \mathrm{e}, \mathrm{e}^{\prime}: \text { Event starts }\left(\mathrm{e}, \mathrm{e}^{\prime}\right) \leftrightarrow\left(\text { begin-point }(\mathrm{e})=\operatorname{begin}-\operatorname{point}\left(\mathrm{e}^{\prime}\right)\right) \wedge \\
\text { precedes }\left(\mathrm{end}-\operatorname{point}(\mathrm{e}), \text { begin-point }\left(\mathrm{e}^{\prime}\right)\right)\end{array}$ \\
\hline T11 & $\begin{array}{c}\forall \mathrm{e}, \mathrm{e}^{\prime}: \text { Event during }\left(\mathrm{e}, \mathrm{e}^{\prime}\right) \leftrightarrow \leftrightarrow \operatorname{precedes}\left(\text { begin-point }\left(\mathrm{e}^{\prime}\right), \text { begin-point }(\mathrm{e})\right) \wedge \\
\text { precedes }\left(\mathrm{end}-\operatorname{point}(\mathrm{e}), \text { begin-point }\left(\mathrm{e}^{\prime}\right)\right)\end{array}$ \\
\hline T12 & $\begin{array}{c}\forall \mathrm{e}, \mathrm{e}^{\prime}: \text { Event finishes }\left(\mathrm{e}, \mathrm{e}^{\prime}\right) \leftrightarrow \text { precedes }\left(\text { begin-point }\left(\mathrm{e}^{\prime}\right), \text { begin-point }(\mathrm{e})\right) \wedge \\
\left(\text { end-point }(\mathrm{e})=\text { begin-point }\left(\mathrm{e}^{\prime}\right)\right)\end{array}$ \\
\hline T13 & $\begin{array}{c}\forall \mathrm{e}, \mathrm{e}^{\prime}: \text { Event equals }\left(\mathrm{e}, \mathrm{e}^{\prime}\right) \leftrightarrow\left(\text { begin-point }(\mathrm{e})=\text { begin-point }\left(\mathrm{e}^{\prime}\right)\right) \wedge \\
\left(\mathrm{end}-\text { point }(\mathrm{e})=\text { begin-point }\left(\mathrm{e}^{\prime}\right)\right)\end{array}$ \\
\hline T14 & $\begin{array}{c}\forall \text { e, } \mathrm{e}^{\prime}: \text { Event has-part }\left(\mathrm{e}, \mathrm{e}^{\prime}\right) \rightarrow\left(\left(\text { begin-point }(\mathrm{e})=\text { begin-point }\left(\mathrm{e}^{\prime}\right)\right) \vee\right. \\
\left.\operatorname{precedes}\left(\text { begin-point }(\mathrm{e}), \text { begin-point }\left(\mathrm{e}^{\prime}\right)\right)\right) \wedge \\
\left(\left(\mathrm{end}-\operatorname{point}(\mathrm{e})=\text { end-point }\left(\mathrm{e}^{\prime}\right)\right) \vee \operatorname{precedes}\left(\text { end-point }\left(\mathrm{e}^{\prime}\right), \text { end-point }(\mathrm{e})\right)\right)\end{array}$ \\
\hline
\end{tabular}




\subsection{World Changes and Situations}

Events are transformations from a portion of reality to another, i.e., they may change reality by changing the state of affairs from one situation to another. The notion of situation employed here is akin to notion of state of affairs in the philosophical literature. However, unlike state of affairs, situations are bound to specific time points. So, two qualitatively indistinguishable situations occurring at different time points are considered as numerically distinct (e.g., the situation of "John having $38^{\circ} \mathrm{C}$ of fever now" and "John having $38^{\circ} \mathrm{C}$ of fever in some moment in the past". A situation is a particular configuration of a part of reality which can be understood as a whole. Situations can be factual or counterfactual (e.g., the situation in which "Al Gore is the president of the USA"). Factual situations are termed Facts [3]. Facts are situations which are said to obtain at particular time points.

We postulate two possible relations between situations and events: (i) a situation $\mathrm{s}$ triggers an $e$ event, in the case that $e$ occurs because of the obtaining of s, and; (ii) an event brings about a situation s, in which case the occurrence of an event $e$ results in the situation $s$ obtaining in the world at the time point end-point(e), i.e., results in $s$ becoming a fact in end-point(e). A triggers relation between situation $s$ and event $e$ captures the notion that $\mathrm{s}$ exemplifies a state of the world that satisfies all the sufficient and necessary conditions for the manifestation of $e$.

A situation that triggers an event obtains at the begin point of that event (S1). A situation brought about by an event obtains at the end point of that event (S2). There is a unique situation that triggers a particular event occurrence (S3) ${ }^{2}$. We also define that there is a unique (maximal) situation that is brought about by an event (S4), embodying the effects of the event at the moment it ends. A fact is a situation which eventually obtains (S5).

\begin{tabular}{|l|c|}
\hline S1 & $\forall$ s:Situation, e:Event triggers(s,e) $\rightarrow$ obtainsIn(s, begin-point $(\mathrm{e}))$ \\
\hline S2 & $\forall$ s:Situation, e:Event brings-about $(\mathrm{e}, \mathrm{s}) \rightarrow$ obtainsIn(s, end-point $(\mathrm{e}))$ \\
\hline S3 & $\forall \mathrm{e}:$ Event $\exists ! \mathrm{s}:$ Situation triggers $(\mathrm{s}, \mathrm{e})$ \\
\hline S4 & $\forall \mathrm{e}:$ Event $\exists ! \mathrm{s}:$ Situation brings-about $(\mathrm{e}, \mathrm{s})$ \\
\hline S5 & $\forall \mathrm{s}:$ Situation fact $(\mathrm{s}) \leftrightarrow \exists \mathrm{t}:$ TimePoint obtainsIn(s,t) \\
\hline
\end{tabular}

Suppose we have a fact $\mathrm{f}$ that is brought about by event e. Now, suppose that $\mathrm{f}$ triggers event e'. In this case, we can state that the occurrence of e' is caused by the occurrence of e. In other words, we can state that e directly-causes e' iff:

\begin{tabular}{|l|c|}
\hline S6 & $\forall \mathrm{e}, \mathrm{e}^{\prime}:$ Event directly-causes $\left(\mathrm{e}, \mathrm{e}^{\prime}\right) \leftrightarrow$ \\
\end{tabular}

Finally, we define a causes (S7) relation between events as follows:

\begin{tabular}{|c|c|}
\hline S7 & 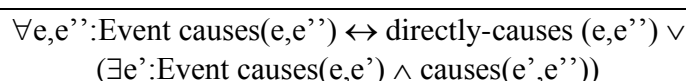 \\
\hline
\end{tabular}

\footnotetext{
${ }^{2} \mathrm{~A}$ situation could be part of other (complex) situations. We refrain from discussing issues regarding the mereology of situations; this would be part of a full theory of situations which is outside the scope of this paper.
} 
Given our characterization of temporal intervals, as well as formulae S1-S7 above, we can demonstrate that causes is a strict partial order. Firstly, given that situations are bound to time points, we can easily show that, given three different events e,e' and $\mathrm{e}^{\prime \prime}$, if we have directly-causes(e,e') and directly-causes( $\left(e^{\prime}, e^{\prime \prime}\right)$, we cannot have that directly-causes(e,e'). The restricted form of causation defined in S7 is also considered here to be a transitive relation. Secondly, the irreflexivity of this relation is rather straightforwardly shown from the constraint that a situation can only be bound to one time point plus the constraint that the two external time boundaries of an event are necessarily distinct and strictly ordered (as in [15], we consider here that there are no zero length events). Moreover, given this constraint, it is easy to show that the causes relation is asymmetric. Finally, one can show that, given distinct events e, e' and e', if directly-causes(e,e') and directly-causes(e',e' '), then we have that e and e' meet (as well as e' and e'') but also that e before e'.

A fragment of UFO-B summarizing the discussion in this section is depicted in Figure 4 below.

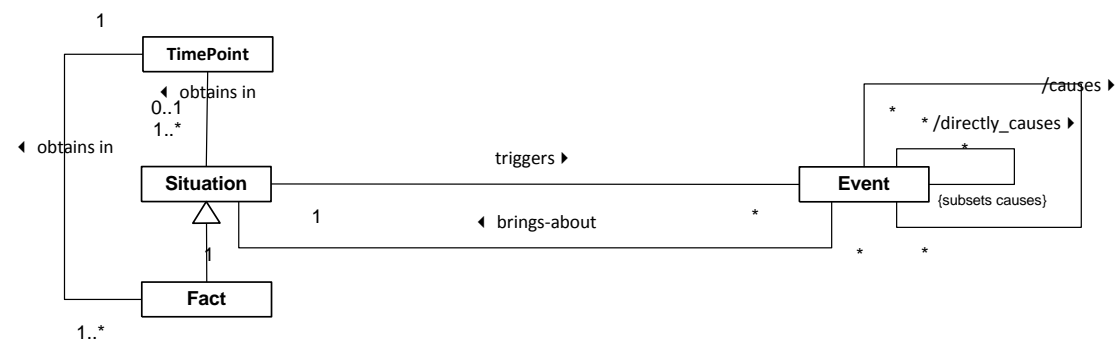

Fig.4. Situations as parts of a world that obtain in particular time points; events as changes.

\subsection{Events as Manifestations of Object Dispositions}

Since its initial versions, UFO's notion of particularized tropes includes both qualities (e.g., color, weight, temperature, electric charge) and dispositions (e.g., the fragility of a glass, the disposition of a magnet to attract metallic material) [3]. Following [11], we consider dispositions as properties that are only manifested in particular situations and that can also fail to be manifested. When manifested, they are manifested through the occurrence of events. Take for example the disposition of a magnet $m$ to attract metallic material. The object $m$ has this disposition even if it is never manifested, for example, because it is never close to any magnetic material. Nonetheless, $m$ can certainly be said to possess that intrinsic (even essential, in this case) property, which it shares with other magnets. Now, a particular metallic material has also the disposition of being attracted by magnets. Given a situation in which $\mathrm{m}$ is in the presence of a particular metallic object (at a certain distance, of a certain mass, in a surface with a certain friction, etc.), the dispositions of these two entities (metallic object, magnet) can be manifested through the occurrence of a complex event, namely, the movement of that object towards the magnet.

The following constraints hold for the view of dispositions assumed here. Firstly, we specify that, as other particularized properties (tropes), dispositions are existentially dependent and therefore inhere in particular objects (D1). Moreover, the events we consider in this paper are manifestations of dispositions (D2). A situation triggers an 
event when this situation activates the disposition that is manifested by that event (D3). Finally, given these assumptions, we have that a particular atomic event is existentially dependent on that particular object because that event is a manifestation of a disposition of that object (which, like any intrinsic property, is entity specific) (D4).

\begin{tabular}{|l|c|}
\hline D1 & $\forall \mathrm{d}$ :Disposition $\exists ! \mathrm{o}:$ Object inheresIn(d,o) \\
\hline D2 & $\forall \mathrm{e}:$ AtomicEvent $\exists$ !d:Disposition manifestedBy(d,e) \\
\hline D3 & $\forall$ s:Situation, e:AtomicEvent triggers(s,e $) \leftrightarrow$ \\
& $\exists$ d:Disposition activates $(\mathrm{s}, \mathrm{d}) \wedge$ manifestedBy(d,e) \\
\hline D4 & $\forall \mathrm{d}:$ Disposition, e:AtomicEvent, o:Object \\
& manifestedBy $(\mathrm{d}, \mathrm{e}) \wedge$ inheresIn $(\mathrm{d}, \mathrm{o}) \rightarrow$ dependsOn $(\mathrm{e}, \mathrm{o})$ \\
\hline
\end{tabular}

Fig. 5 depicts a fragment of UFO-B summarizing the discussions in this section.

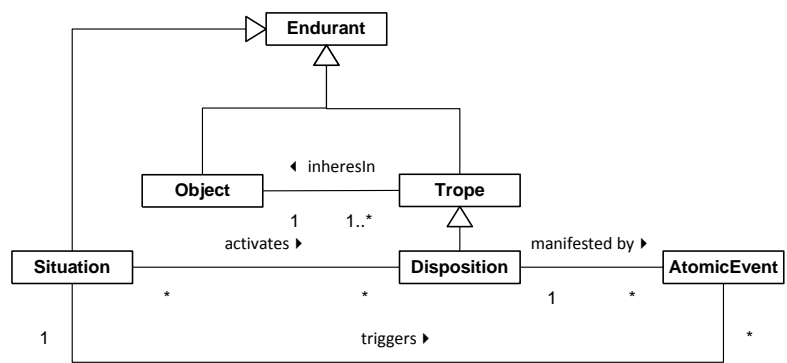

Fig.5. Atomic events as manifestations of object dispositions

\section{Evaluation and Proof of Concept}

The axiomatization of UFO-B presented in this paper has been fully implemented in the formal language Alloy [12] and can be obtained from http://nemo.inf.ufes.br/ufo-

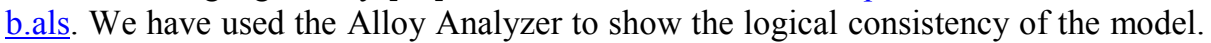
Moreover, in order to evaluate this model, we have employed the approach discussed in [5] of model evaluation via visual simulation. In a nutshell, we have configured a visual profile for UFO-B instances (available at http://nemo.inf.ufes.br/ufo-b.thm). The Alloy Analyzer then generates possible logical instances of this logical theory (given a finite domain of quantification), which are then visualized in the visual profile. By iterating through these visual instances, the modeler can detect the existence of logical models, which describe ontologically inconsistent state of affairs and, hence, rectify the ontology specification at hand.

The extended version of UFO-B reported here has been used in a number of industrial applications in the domain of media content management in a large media conglomerate in Brazil $[16,17]$. In that context, this ontology has been used, among other things, in the construction of an ontology of soccer. That ontology can be used to annotate and reason with specific events taking place inside a game and to organize all the desired statistic information of a game. As reported by the organization's product owner and author of [17], UFO-B played a fundamental role in solving a number of conceptual problems of the original soccer ontology used in that organization and in the production of an ontology which is more truthful to the underlying domain. 


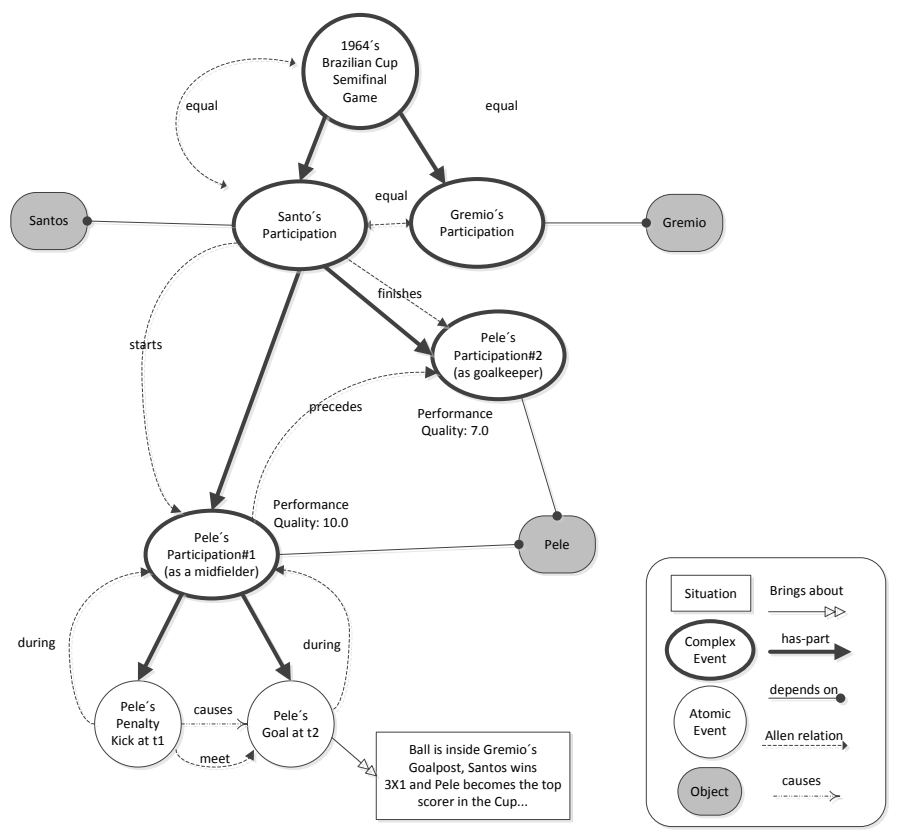

Fig. 6. A fragment of a football game described with UFO-B

The model depicted in Fig. 6 illustrates how UFO-B can be used for describing a historic soccer game. The game is the 1964's Brazilian Cup Semifinal Game between the teams Santos and Gremio, in which Pelé played both as a Midfielder and as a Goalkeeper. The game was composed of two team participations: Santos' participation and Gremio's participation. These two participations were (as always) completely synchronized (started and ended at the same time) and were also synchronized with the game itself. Pelé had two participations in the game: participation\#1 (p1), as an offensive midfielder, and participation\#2 (p2), as the goal keeper. (p1) begins with the beginning of Santos' participation (hence, with the beginning of the game) and stops before the end of the game (we can then infer that Pele did not play the whole game as a Midfielder); (p2) starts in the middle of the game and lasts until the end of the game. As one can observe, there is no temporal overlap between these two participations (one precedes the other). In this game, Pelé's participations as midfielder and goal keeper have been predicated different levels of performance quality: as a midfielder he had a 10.0 performance mark and as a goalkeeper he had a 7.0 mark. Pelé's participation\#1 is composed of other sub-events (which are part of Santos' participation and part of the game, transitively). Two of these events are Pelé's Penalty Kick at a certain time $\mathrm{t}_{1}$, and Pelé's scoring of a Goal at time $\mathrm{t}_{2}$. These two events are causally related, i.e., it is Pelé's Penalty Kick that causes Pelé's scoring of the Goal. The situation (fact) brought about by the latter event includes changes in properties of a number of entities (e.g., Pelé's number of scores in the cup, the score of the game, the position of the ball). 


\section{Related Work}

The notion of event is present in many upper-level ontologies (e.g., DOLCE [7], BWW [2], Kaneiwa et al [6]), as well as in lightweight semantic web ontologies such as the Event Ontology [10]. Here, due to lack of space, we can compare UFO-B only to a subset of these proposals: the Event Ontology, BWW and Kaneiwa et al.

The BWW ontology is based on the work of the philosopher Mario Bunge [2]. Over the years, it has been employed by a number of authors in the evaluation and redesign of many conceptual modeling languages and reference models. For Bunge, an event is simply defined as a formal relation between two points in the state space of an entity. Although representing an aspect of change, the change is limited to one entity, i.e., it is not the case that an event can have several participants and that individual participations can be reified, predicated upon or further mereologically decomposed. Moreover, pre- and post-states of events (involving multiple entities) cannot be modeled. In other words, one cannot model that it is the same event (e.g., a marriage) that changes the properties of both the husband and the wife. In summary, in BWW, there is also no support for an event mereology, participation differentiation, temporal relations between events and object dispositions.

The Event Ontology is used, for example, by the BBC in the design of a number of domain ontologies (e.g., the Music Ontology and the Sports Ontology ${ }^{3}$ ). This ontology considers events in terms of their participants, their temporal and spatial properties and their composition. The authors do not explain, however, what are the constraints applying to the relation between an event and its proper parts, i.e., no axiomatization for the assumed event mereology is presented. The temporal properties of events are defined via a mapping to OWL Time, which commits the model to a particular ontology of time intervals, but has the advantage of inheriting the basic axiomatization of Allen's relations. Participation of objects in events can be modeled by the sub event relation together with a relation tying those objects to these subevents. This strategy, however, makes it harder to differentiate participations from other mereological parts of events. In summary, the Event Ontology has a limited treatment of mereological relations, a limited treatment of participation, a fixed treatment of temporal properties, no representation for causation and no support for the representation of dispositions and their connection to objects and events. Furthermore, except for the axiomatization of the Allen's time interval relations inherited from OWL Time, no further axiomatization for composition and participation is presented.

In [6], Kaneiwa et al. propose an Upper-Level Ontology of Events. The proposed ontology offers a classification of events according to the nature of their participants, namely, between natural and artificial events. In this approach, events are defined as tuples formed by different types of constituents. For instance, natural events are characterized either by a time and location or an object, time and location. In contrast, artificial events have an agent (individual or collective), a time and a location, or an agent, an object, a time and a location. Regarding event relations, causality is defined in Kaneiwa et al. in two modes: (1) an object can cause an event, and (2) an event can

${ }^{3}$ http://www.bbc.co.uk/ontologies/ 
cause an event. In pace with [4], we reject the former: objects do not cause events; only events cause events (sometimes via the manifestation of the disposition of these objects). The only meta-property of the causation relation put forth by Kaneiwa et al. is transitivity (in accordance with UFO-B). Regarding temporal event relations, the authors seem to consider the relations of precedes, meets, finishes and overlaps, i.e., only a subset of Allen's time interval relations. Finally, regarding parthood, the only formal constraint considered is that the proper parts of an event must be temporally included in its duration (which is also considered in UFO-B).

\section{Practical Implications and Final Considerations}

The view of events put forth in this paper can be summarized as follows: objects have (particularized) properties, some of which are dispositions, which are properties that are manifested in particular situations through the occurrence of events. The atomic events considered here are manifestations of single dispositions; complex events are manifestation of several dispositions. When a complex event is a manifestation of different dispositions of different objects, we can isolate those slices of such an event, which directly depend on one of these objects. We call these slices participations. The participation view of events is in some sense orthogonal to the mereological view. Thus, participations of individual objects in events can both be mereologically simple or complex. In this view, events (as much as objects) can also be predicated with qualitative characteristics. Events are delimited by time boundaries (here, time points which are totally ordered). Thus, events happen in time and several different temporal relations between events can be derived from the ordering of their time boundaries. These events considered here, as manifestations of dispositions, change the world, by mapping one situation to another. Situations that are brought about by the manifestation of dispositions and can activate other dispositions, making the world "tick". The unfolding of the relations between situations, dispositions and events with further activation and manifestation of other dispositions can be used to characterize an admittedly limited but very useful form of (direct and indirect) causation between events.

The work presented here is an extension of our early work on an ontological theory of events as presented in [18]. That preliminary theory of events gave us the opportunity to conduct relevant case studies for analyzing and (re)designing conceptual modeling languages, reference frameworks and domain ontologies in areas such as Enterprise Architecture, Business Process Modeling, Software Engineering, Bioinformatics, Telecommunications, Discrete-Event Simulation, Collaborative Processes, Service Management among others ${ }^{1}$. The significant experience acquired on these projects in different domains over the years played an important role in selecting the type of concepts we believe a foundational ontology for the conceptual modeling of events should address. However, the ontology of events proposed in [18], despite being based on a number of results from the formal ontology literature, was presented in an informal manner. As discussed in [5,6], having an axiomatized formal semantics for a foundational ontology plays a fundamental role in supporting automated reasoning as well as the formal verification and validation of conceptual models and domain ontologies derived from it. The contributions of this paper are then two-fold: (i) first- 
ly, it extends both the width and depth of our previous ontological treatment of events; (ii) secondly, it presents a first comprehensive axiomatization of this ontology.

Regarding (i), the work presented here extends our previous work in important ways. For instance, in [18], we mention that events can be further decomposed in subevents. However, we make no commitment there to a specific mereology of events (extensional mereology). Moreover, in our previous work, there is no discussion on dispositions and their relation to situations and to a form of causation. This extension to the theory makes an important contribution in connecting our ontology of events with our ontology of endurants and, consequently, in supporting a systematic connection between structural and dynamic conceptual models based on these ontologies.

The notions comprising UFO-B have interesting implications to the practice of conceptual modeling. For instance, in [9], Olivé defends the representation of events in structural conceptual models. However, if events are to be represented in structural models, one should also be able to represent parthood relation between events in those models. As demonstrated in [3], parthood relations between objects can exhibit two modes of dependence, namely, generic and existential dependence. For instance, while a person depends generically on an instance of heart (whilst every person must have an instance heart, it does not always have to be the same instance of heart), she depends existentially on a particular individual instance of brain. In [3], these are called mandatory and essential parts, respectively. Now, with the adoption of an extensional mereology for events, we can show that there are no mandatory parts of events (!), i.e., all parts of events are essential parts (M9). As a consequence, whenever representing a parthood relation in a structural conceptual model (e.g., in a UML class diagram), the association end connected to the part must be deemed immutable (readOnly). In the same spirit, given the notion of participation presented here, whenever representing events in a structural conceptual model, these events should be connected to their participants via an existential dependence relation (entailing an immutability constraint in the association end connected to the types representing each participant). Moreover, the fragments of the UFO-B ontology as presented here (figures 2-5) can be re-used as ontology design patterns to address recurrent modeling problems. For instance, in UML class diagrams, one can represent that an event (e.g., a musical session) can be composed of zero-to-many subevents. Now, if an event can be composed of zero-to-many subevents, then it can be composed of one unique subevent. But what then is the difference between a musical session and the unique subsession that composes it? This situation is prevented by the (M6) axiom in UFO$\mathrm{B}$ : a musical session (business process) is either atomic or it is composed of at least two disjoint events. From a modeling perspective, this can be addressed by a direct instantiation of the model fragment in figure 2 and its associated formal constraints.

The ontological theories comprising UFO are well known and supported in the philosophical literature. For instance, our mereology of events corresponds to the standards treatment of events in the philosophical literature [13]; the notion of participation is articulated here as the perdurant counterpart of the notion of qua individuals (or role instances) [3]; the treatment of temporal ordering of events adopted here (Allen's Relations) is also well known and adopted [14]. Finally, the use of theory dispositions as tropes to articulate the relation between situations and causation also finds strong support in the formal ontology literature [11]. One of the key contributions of 
UFO-B is to extend a combination of existing results from formal ontology in a fuller theory for supporting the foundations of events in conceptual modeling.

It is important to highlight that what is presented in this paper is a proper fragment of the entire extended axiomatized UFO-B ontology. In particular, due to space limitations, we have left out sub-theories dealing with: (i) the differentiation of roles played by objects inside an event (the so-called processual roles); (ii) qualities and quality structures used to predicate qualitative aspects to events; (iii) particular aspects of events of creation, destruction and modification. These theories shall be presented in an extension of this paper. Nonetheless, the interested reader can find them in the Alloy axiomatization of UFO-B available in http://nemo.inf.ufes.br/ufo-b.als.

Acknowledgements. This work has been supported by FAPES (PRONEX Grant \#52272362/2011). We thank Nicola Guarino for fruitful discussions and comments.

\section{References}

1. Santos Junior, P. S. et al., An Ontology-Based Analysis and Semantics for Organizational Structure Modeling in the ARIS Method. Information Systems (Oxford), 2013.

2. Weber, R., Ontological Foundations of Information Systems, Coopers \& Lybrand, 1997.

3. Guizzardi, G., Ontological Foundations for Structural Conceptual Models, Telematics Institute Fundamental Research Series, n. 15, University of Twente, The Netherlands, 2005.

4. Galton, A., States, Processes and Events, and the Ontology of Causal Relations. 7th International Conf. on Formal Ontology in Information Systems (FOIS 2012), Graz, 2012.

5. Braga, B.F.B. et al.: Transforming OntoUML into Alloy: towards conceptual model validation using a lightweight formal method, ISSE, ISSN 1614-5046, vol. 6, Springer, 2010.

6. Kaneiwa, K.; Iwazume, M.; Fukuda, K., An Upper Ontology for Event Classifications and Relations, 20th Australian joint conference on Advances in artificial intelligence, 2007.

7. Masolo, C., Borgo, S., Gangemi, A., Guarino, N., Oltramari, A. 'Ontology Library', WonderWeb Deliverable D18, 2003.

8. Herre, H., General Formal Ontology (GFO): A Foundational Ontology for Conceptual Modelling, Handbook of Theory and Application of Ontologies, Springer-Verlag, 2010.

9. Olivé, A.; Raventós, R., Modeling events as entities in object-oriented conceptual modeling languages, Data \& Knowledge Engineering 58 (2006) 243-262.

10. Raimond, Y., Abdallah, S., The Event Ontology, http://motools.sourceforge.net/event/event.html, 2007.

11. Mumford, S., Dispositions, Oxford University Press, 2003.

12. Jackson, D., Software Abstraction: Logic, Language and Analysis, MIT Press, 2012.

13. Simons, P., Parts: A Study in Ontology, Oxford University Press, 1997.

14. Allen, J.F., Maintaining Knowledge about Temporal Intervals, Communications of the ACM, Vol.26, no. 11, 1983

15. Linehan, M., Barkmeyer, E., Hendryx, S., The Date-Time Vocabulary, 7th International Conference on Formal Ontology in Information Systems (FOIS 2012), Graz, 2012.

16. Carolo, F.P.; Burlamaqui, L., Improving Web Content Management with Semantic Technologies, Semantic Technology and Business Conference (SemTech 2011), San Francisco.

17. Pena, R., Semantic Support for Publishing Journalistic Content on the Web (in portuguese), MSc dissertation, Pontifical Catholic University, Rio de Janeiro, Brazil, 2012.

18. Guizzardi, G., Falbo, R. A., and Guizzardi, R. S. S. Grounding software domain ontologies in the Unified Foundational Ontology (UFO): The case of the ODE software process ontology, 11th Iberoamerican Conference on Software Engineering. pp. 244-251, 2008. 